\title{
4. Organoide: Ein wesentliches Element in einem generativen Modellgefüge
}

Übersetzt von Anja Pichl

\subsection{Einleitung}

In diesem Kapitel werden konzeptuelle Fragen der Organoidforschung aus einer wissenschaftsphilosophischen Perspektive erörtert. Im Kern besteht Philosophie in der Tätigkeit des abstrakten Nachdenkens über allgemeine Fragestellungen. Daher gibt es keine scharfe Trennlinie zwischen Philosophie und anderen Arten der Nachforschung - einschließlich der Naturwissenschaften. Die Wissenschaftsphilosophie ist ein eigenständiger Teilbereich der Philosophie, der argumentative und analytische Methoden der Philosophie zur Untersuchung der Wissenschaften nutzt. Ziel ist die Klärung zentraler Fragen, die meist im Hintergrund wissenschaftlicher Arbeiten stehen. Dazu gehören Fragen nach dem Wesen, der Struktur und der Grundlage wissenschaftlichen Wissens, den Methoden zum Erwerb solchen Wissens und der Bedeutung wissenschaftlichen Wissens für die Gesellschaft im weiteren Sinne. Insofern ist das Anliegen dieses Themenbands, den Stand der Organoidforschung darzustellen und ihre Methoden und möglichen Ergebnisse zu reflektieren, teilweise philosophischer Natur. Dieses Kapitel nutzt Erkenntnisse der gegenwärtigen wissenschaftsphilosophischen Forschung, um die Bedeutung und Rolle von Organoiden in den Naturwissenschaften zu klären, d. h. die Art und Weise wie sie zu wissenschaftlichem Wissen beitragen.

Organoide sind vereinfachte Annäherungen an Organe, die durch das „Aussäen“ von Stammzellen in eine dreidimensionale Kultur hergestellt werden, und zwar unter biochemischen Bedingungen, die auf die Entwicklung eines bestimmten Organs abgestimmt sind. ${ }^{1}$ Ihre vorrangige wissenschaftliche Funktion ist die von Modellen menschlicher Organe; sie sind aus Stammzellen hergestellte Modellorgane. Wissenschaftliche Modelle und Modellierungen sind zentrale Themen der gegenwärtigen

1 Für detaillierte Darstellungen des Herstellungsprozesses verschiedener Organoide siehe auch Kap. 3. 
Wissenschaftsphilosophie. Deshalb können Erkenntnisse der Wissenschaftsphilosophie einige der Fragen klären, die das große Ganze der Organoidforschung betreffen, und so die Erkenntnisse der naturwissenschaftlichen Experten ergänzen. In Abschnitt 4.2 wird ein einflussreiches philosophisches Konzept von Modellen vorgestellt und auf die Organoidforschung angewendet. Mithilfe dieses Konzeptes lässt sich klären, wie Ähnlichkeiten zwischen Organoiden und den Organen, denen sie ähneln, Rückschlüsse auf Letztere stützen und inwiefern die Modellfunktion von Organoiden diesen Ähnlichkeiten Grenzen setzt. In Abschnitt 4.3 wird das philosophische Konzept auf lebende Modelle ausgeweitet und argumentiert, dass die materielle Kontinuität von Organoiden mit menschlichen Stammzellen in Verbindung mit deren Entwicklungsfähigkeiten modellbasierte Schlussfolgerungen über die Ziele von Organoiden absichert. Ein weiteres Ergebnis ist, dass Organoide als Teil eines breiteren ,Gefüges von Modellen' („fabric of models") konzeptualisiert werden sollten, die eine materielle Kontinuität sowohl mit anderen Modellen als auch mit ihren Zielen aufweisen. Aufbauend auf diesen Ergebnissen wird in Abschnitt 4.4 geklärt, in welchem Sinn Organoide sich „selbst organisieren“. Im Kontext der Organoidforschung sollte der Begriff „Selbstorganisation“ von Wissenschaftlerinnen und Wissenschaftlern im Gegensatz zur experimentellen Kontrolle verstanden werden, und nicht in dem Sinne, als verfügten Stammzellen über ein inneres, vorgeformtes Programm zur Organentwicklung. In Abschnitt 4.5 werden die Schlussfolgerungen vorgestellt.

\subsection{Organoide als wissenschaftliche Modelle: eine philosophische Analyse}

Organoide fungieren in erster Linie als Modelle für menschliche Organe. Was bedeutet das? Die Grundidee ist in die Methode zur Herstellung von Organoiden eingebaut. Organoide werden aus Stammzellen hergestellt. Sofern die Mikroumgebung (oder Nische) von Stammzellen es zulässt, differenzieren diese zu reiferen Zellen. Zur Mikroumgebung gehören auch bestimmte räumliche Beziehungen der Stammzellen zueinander. Die Stammzellen interagieren und bringen so eine Annäherung (,approximation") an ein Organ hervor. ${ }^{2} \mathrm{Zu}$ den auf diese Weise approximierten Organen gehören der Sehnervenkelch, mehrere Gehirnregionen (siehe Tanaka/Park, Kap. 3.5), der Darm (siehe Interview mit Clevers, Kap. 2.2 und Kayisoglu/Schlegel/Bartfeld, Kap. 3.8), die Leber, die Nieren (siehe Gupta/Dilmen/Morizane, Kap. 3.6), der Magen und die Bauch-

2 „Annäherung“ (,approximation“) stammt aus dem Titel der Sonderausgabe über Organoide der Zeitschrift Science von 2019. 
speicheldrüse. Die Annäherung umfasst kompositionelle, strukturelle und funktionelle Aspekte: mehrere organspezifische Zelltypen; relative Positionen von Zelltypen, die in mancher Hinsicht dem vollentwickelten Organ ähneln; eine organspezifische Funktion wie z. B. neuronale Aktivität in Hirnorganoiden oder Verdauungsenzyme in Darmorganoiden. Diese kurze Skizze wirft mehrere Fragen auf. Wie führt eine Annäherung in diesem Sinne zu wissenschaftlichem Wissen? Wissen worüber genau? Wo liegen die Grenzen und Stärken von „Wissen durch Annäherung“? Philosophische Darstellungen von wissenschaftlichen Modellen und Modellierungen geben Antworten auf diese und verwandte Fragen.

Obgleich Philosophinnen und Philosophen viele Aspekte wissenschaftlicher Modelle und Modellierung kontrovers diskutieren, besteht ein breiter Konsens in einigen wesentlichen Punkten:

- Modelle sind auf ein Ziel ausgerichtet. Ein Modell existiert nicht für sich allein; es muss Modell von etwas anderem als es selbst sein (Keller, 2000). Ein Modell zu sein ist relational und diese Beziehung ist vom Modell auf sein Ziel hin ausgerichtet.

- Die Verwendung von Modellen ist intentional. Es reicht nicht aus, zu beschreiben, wie ein Modell sich zu seinem Ziel verhält, denn die wissenschaftliche Funktion eines Modells steht im Verhältnis zu den Intentionen und Zwecken seiner Nutzerinnen und Nutzer und wird zum Teil durch diese bestimmt (siehe Giere, 1988; Frigg/Nguyen, 2018; Boesch, 2019 und dortige Referenzen). Um Erstere zu verstehen, muss man sich mit Letzteren befassen.

- Modelle können abstrakt oder konkret sein. Es besteht eine große ontologische Vielfalt in der Klasse der Dinge, die als Modelle fungieren: Gleichungen, abstrakte imaginäre Objekte (z. B. unendliche Populationen, perfekte Sphären), maßstabsgetreue Modelle, computergenerierte Visualisierungen und sogar ganze Organismen (Weisberg, 2013).

- Modelle stützen Rückschlüsse auf ihre Ziele. Das bedeutet, dass Modelle eine Beweisquelle sind - aber eine indirekte. ${ }^{3}$ Direkte Evidenz besteht aus Beobachtungen eines Zielsystems, des Phänomens, das wir verstehen wollen. Aber Modelle sind verschieden von ihren Zielen. Deshalb ist es wichtig, zu zeigen, dass die Schlussfolgerungen, die sie stützen, zuverlässig sind. Aufgrund der Verschiedenheit wissen-

3 Godfrey-Smith (2006), Weisberg (2013) und andere definieren ein Modell als Repräsentation eines komplexeren, detaillierteren und facettenreicheren Ziels. Das Modell wird ihnen zufolge von Wissenschaftlerinnen und Wissenschaftlern im Einklang mit Standards konstruiert, die sich an seinem intendierten Zweck orientieren. Ob alle Modelle der Repräsentation dienen, wird heiß diskutiert. Ihre Schlussfolgerungen stützende Rolle ist hingegen allgemein anerkannt. 
schaftlicher Modelle ist es schwierig, allgemeine Prinzipien für zuverlässige modellbasierte Schlussfolgerungen zu formulieren. Um die Verlässlichkeit der durch ein Modell gestützten Schlussfolgerungen zu beurteilen, müssen wir sowohl die Modell-Ziel-Beziehung als auch den Zweck, für den das Modell konstruiert wurde, genau betrachten. Was das Erstere betrifft, so werden modellbasierte Schlussfolgerungen häufig mit den Ähnlichkeiten zwischen Modell und Ziel begründet. ${ }^{4}$

Fügt man diese Überlegungen zusammen, so ergibt sich Folgendes: Modelle sind Werkzeuge für stellvertretendes Denken (,surrogate reasoning“5) in Bezug auf Ziele, deren Erfolgskriterien von den Zwecken des Modellnutzers abhängig sind. Der Gebrauch jeglichen Modells kann als vierstellige Beziehung zwischen einem Handelnden, einem Modell, einem Ziel und einem Zweck analysiert werden: „der Handelnde (1) intendiert; (2) ein Modell M zu verwenden; (3) um einen Teil der Welt W darzustellen; (4) für die Zwecke Z. Dementsprechend spezifizieren die Handelnden, welche Ähnlichkeiten intendiert sind und zu welchem Zweck“ (Giere, 2010: 274). Dieses Verständnis von Modellen trägt zur Klärung des Wesens und der Verwendung von Organoiden bei. ${ }^{6}$

Die Arbeitsdefinitionen des Begriffs „Organoid“ in der wissenschaftlichen Literatur betonen deren Struktur und Funktion, ihren Ursprung in Stammzellen und ihre hauptsächliche Verwendung. So werden Organoide beispielsweise definiert als „3-D-Kulturen, die zur Untersuchung der Organbildung ex vivo verwendet werden“ (Munsie et al., 2017: 942), als „dreidimensionale, multizelluläre, aus Stammzellen gewonnene Konstrukte, die In-vivo-Gewebe nachahmen“ (Nature Methods, 2018: 1) und als „dreidimensionale Strukturen mit multizellulärer Komplexität und einem gewissen Grad an Gewebestruktur und -funktion“ (Takebe/Wells, 2019: 956). Die detaillierteste und systematischste Definition, die mir bekannt ist, findet sich bei Lancaster und Knoblich (2014). ${ }^{7}$ Ihre Darstellung enthält die in anderen Arbeitsdefinitionen betonten Merkmale und geht darüber hinaus auf essenzielle Merkmale von Organoiden ein: „Ein

4 Hierdurch gehören modellbasierte Schlussfolgerungen zu den Analogie-Argumenten (,argument from analogy“) - eine Form des induktiven Argumentierens, das sich formaler Analyse entzieht. Die klassische philosophische Arbeit zu diesem Thema ist Hesse (1966).

5 Dass Modelle Werkzeuge für „stellvertretendes Denken“ sind, bedeutet, dass sie ein Ersatzsystem oder stellvertretendes Untersuchungsobjekt (z. B. ein Hirnorganoid) darstellen, anhand dessen Erkenntnisse über das eigentliche, ggf. nicht gut zugängliche, Objekt des Forschungsinteresses (z. B. das Gehirn) gewonnen werden können.

6 Ich behaupte nicht, dass Gieres Analyse für alle (wissenschaftlichen) Modelle richtig ist, sondern beschränke mich auf die Aussage, dass sie hilfreich ist für die Klärung der Modellfunktion von Organoiden.

7 Siehe Simian und Bissell (2017) für eine wichtige abweichende Sichtweise. 
Organoid ist eine Ansammlung organspezifischer Zelltypen, die sich aus Stammzellen oder Vorläuferzellen von Organen entwickeln und sich selbst organisieren - durch Zellsortierung und räumlich begrenzte Zelldifferenzierung in ähnlicher Weise wie in vivo“ (1247125-1). Diese Definition basiert auf drei Voraussetzungen, die Lancaster und Knoblich zufolge erfüllt sein müssen, damit eine aus Stammzellen gewonnene dreidimensionale Kultur als Organoid bezeichnet werden kann. Die kultivierte Entität muss:

1) mehrere für das reife Organ charakteristische Zelltypen enthalten,

2) mindestens eine organspezifische Funktion erfüllen und

3) eine zelluläre Organisation (räumliche Anordnung) aufweisen, die in mancher Hinsicht der des reifen Organs ähnelt.

So definiert sind Organoide Produkte von In-vitro-Entwicklungsprozessen, die durch Stammzellen initiiert werden. Zudem sind sie (teilweise) zusammengesetzt aus mehreren organspezifischen Zelltypen, die so organisiert sind, dass sie mindestens eine organspezifische Funktion erfüllen können (z. B. neuronale Aktivität, Entgiftung durch Hepatozyten [Leberzellen], Magensäuresekretion). Die erforderlichen Ähnlichkeiten im Hinblick auf Zusammensetzung, Funktion und räumliche Anordnung lassen sich messen, indem man das Organoid mit dem jeweiligen reifen menschlichen Organ vergleicht. Diese Ähnlichkeiten werden als Beweis für weitere Ähnlichkeiten herangezogen, wie z. B. zelltypspezifische Genexpressionsmuster, Entwicklungslinien, ZellZell-Interaktionen und Zell-Matrix-Interaktionen. Diese und andere abgeleitete Ähnlichkeiten basieren auf der allgemeinen Hypothese, dass der Entwicklungsprozess, der ein Organoid hervorbringt, der Entwicklung in vivo ähnelt (die im Menschen nicht beobachtet werden kann). Auf diese Weise sind Organoide Surrogat-Forschungsobjekte, die an die Stelle unzugänglicher Prozesse der menschlichen Organentwicklung treten, wobei sich diese Rolle auf beobachtbare Ähnlichkeiten stützt.

Dieses Verständnis der wissenschaftlichen Funktion von Organoiden passt sehr gut zum philosophischen Theorierahmen für die Analyse von Modellen. Die Handelnden sind offensichtlich Wissenschaftlerinnen und Wissenschaftler, die Organoide herstellen und verwenden. Gleichermaßen offensichtlich ist, dass Organoide konkrete, materielle Modelle sind. Ihre Ziele sind menschliche Organe in vivo, wobei ein Organ oder ein Teil eines Organs jeweils einer Art von Organoid entspricht. Tatsächlich sind die Nomenklatur und Klassifikation von Organoiden bezeichnend für ihre Modellierungsfunktion: Organoide werden nach ihren In-vivo-Gegenstücken benannt und unterschieden. Ihre gezielte Ausrichtung auf ein menschliches Organ ist insofern explizit und zentral. Ähnlichkeiten zwischen Modell und Ziel sind essenziell; 
Organoide werden so konstruiert, dass sie einem Zielorgan möglichst ähnlich sind. ${ }^{8}$ Die Verwendungszwecke von Organoiden sind vielfältig; sie reichen von einem besseren Verständnis der menschlichen Embryonalentwicklung über patientenspezifische Krankheitsmodelle bis hin zu einem effizienten Screening in der Medikamentenentwicklung. ${ }^{9}$ Das Hauptziel ihrer Anwendung ist jedoch, Wissen über die In-vivo-Entwicklung und -Erhaltung bestimmter menschlicher Organe (z. B. Leber, Darm, Gehirn) sowie damit in Zusammenhang stehende Phänomene zu erlangen (siehe auch Frum/ Spence, Kap. 3.1) - ihre anderen Zwecke basieren weitgehend auf dieser Funktion. Wissenschaftlerinnen und Wissenschaftler gehen davon aus, dass Organoide die Prozesse der normalen menschlichen Organentwicklung auf Zellebene imitieren („rekapitulieren“). Diese Prozesse auf Zellebene werden als "Selbstorganisation“ charakterisiert, die Lancaster und Knoblich (2014) weiter analysieren als Zellsortierung und räumlich begrenzte Festlegung auf bestimmte Zellidentitäten. Organoide bieten einen noch nie dagewesenen Zugang zur Beobachtung und experimentellen Untersuchung dieser und weiterer Prozesse, die an der Bildung und Aufrechterhaltung bestimmter menschlicher Organe beteiligt sind. Der Analogieschluss ist insbesondere für das Verständnis menschlicher Organe mit phylogenetisch einzigartiger Struktur und/oder Funktion, wie z. B. das Gehirn oder die Entgiftungsmechanismen der Leber, von großem Wert.

Wenn diese Darstellung richtig ist, dann gewähren Organoide nur insofern Wissen über die menschliche Organentwicklung in vivo, als indirekte, modellbasierte Schlussfolgerungen zuverlässig sind. Die Zuverlässigkeit dieser indirekten Rückschlüsse beruht auf Ähnlichkeiten zwischen Organoiden und den Organen, denen sie sich annähern (,approximieren“). Daher scheint es, dass Organoide ihren Zielorganen so ähnlich wie möglich sein sollten - je größer die Ähnlichkeit zwischen Modell und Ziel,

8 Je nach Zweck kann das Ziel variieren, vom Menschen im Allgemeinen bis hin zu einem bestimmten Patienten oder (editiertem) Genom.

9 Im Editorial von Nature Methods (2018), in dem Organoide als Methode des Jahres 2017 präsentiert werden, werden sechs Anwendungsmöglichkeiten unterschieden: (1) Zugang zur vorgeburtlichen Entwicklung und Gewebeversorgung des Menschen; (2) Quelle von Gewebe, um viele bisher unzugängliche Phänomene der menschlichen Entwicklung zu studieren; (3) Krankheitsmodelle für Entwicklungsstudien, personalisierte Medizin, Strategien der Krebsbekämpfung; (4) Genome-Editing zur Untersuchung des Verhältnisses von Genotyp und Phänotyp; (5) vergleichende Studien zur Evolution von Primaten; (6) experimenteller Zugang zum einzigartigen menschlichen Gehirn. Hinzufügen ließen sich: Screenings auf Medikamentenwirksamkeit und Toxizität; prä-klinische Forschung und autologer Organersatz. 
desto besser. ${ }^{10}$ In jüngsten wissenschaftlichen Kommentaren zu Organoiden werden spezifische Unähnlichkeiten als Herausforderungen identifiziert, die es zu überwinden gilt:

- Organoide enthalten nicht alle Zelltypen des entsprechenden Organs; nur einige.

- Organoide erfüllen nicht alle Funktionen des vollentwickelten Organs; lediglich einige Aspekte von einigen Funktionen.

- Organoide sind wesentlich kleiner als die Organe, die sie repräsentieren.

- Organoiden fehlt die strukturelle Komplexität des entsprechenden vollentwickelten Organs (Zellanordnungen).

Experten schlagen Wege vor, wie diese Diskrepanzen überwunden werden könnten. ${ }^{11}$ So könnte beispielsweise eine zunehmende Größe und Komplexität durch Vaskularisation (Gefäßversorgung) oder Innervation (Versorgung mit Nerven) erlangt werden, indem technisch hergestellte Pendants von Blutgefäßen oder neuronalen Verbindungen zu einem sich entwickelnden Organoid hinzugefügt werden. Im gesamten Organismus wirkende, homöostatische (die Funktion aufrechterhaltende) und andere Rückkopplungsmechanismen könnten dadurch nachgeahmt werden, dass Multi-Organoid-Systeme zu einem einzigen integrierten Modell verbunden werden. Komplizierte Muster der Zell-Zell-Kommunikation, die die Organentwicklung in vivo in dynamischer Weise koordinieren, könnten in Organoiden durch spezifische Kombinationen und zeitliche Abstimmung biochemischer Signale nachgeahmt werden. In Anbetracht der Tatsache, dass „Gewebeentwicklung, Komplexität, Funktion und Reife eng miteinander verbunden sind“ (Takebe/Wells, 2019: 957), könnte es den Anschein haben, dass Fortschritte in der Organoidforschung zu immer besseren Annäherungen an das Original führen und in perfekten Nachbildungen des Organs kulminieren sollten - reif für die autologe Transplantation.

Allerdings gibt es wichtige konzeptuelle Gründe, dieser „monotonen“ Sichtweise auf Fortschritt in der Organoidforschung zu widerstehen. Damit soll weder behauptet

10 Unähnlichkeit zwischen einem Organoid und seinem Zielorgan ist nicht dasselbe Problem wie die Variabilität unter Organoiden, die aus denselben Stammzellen unter denselben Kulturbedingungen hergestellt worden sind. Die beiden Probleme hängen jedoch zusammen. Insofern als menschliche Organe in vivo nicht dieselben Variationsmuster wie die ihnen korrespondierenden Organoide aufweisen, läuft dies auf eine weitere Unähnlichkeit hinaus: variable Organoide im Unterschied zu nicht variablen Organen (im Hinblick auf manche Züge oder Merkmale). Die Variabilität unter Organoiden im Hinblick auf ein bestimmtes Merkmal (z. B. Größe oder Form) könnte jedoch eliminiert werden, während die Verschiedenheit vom Zielorgan bestehen bleibt.

11 Siehe z. B. Yin et al. (2016), Park et al. (2019), Takebe/Wells (2019). 
werden, dass größere Ähnlichkeiten unerreichbar, noch dass auf Organoiden basierende Hoffnungen auf regenerative Medizin vergeblich seien. Jedoch sollte die Organoidforschung nicht so verstanden werden, als ziele sie ausschließlich auf perfekte strukturelle und funktionelle Kopien menschlicher Organe ab, die Entwicklungsprozesse in vivo exakt nachbilden. Perfekte Ähnlichkeit kann nicht das Ziel (oder der Maßstab) der Organoidforschung sein, weil kein Modell mit seinem Ziel identisch ist. Modelle sind keine Kopien. Wenngleich Ähnlichkeiten zentral sind (zumindest für die Klasse von Modellen, zu denen Organoide gehören), unterscheiden sich Modelle auch von ihrem Ziel in bestimmter Weise. ${ }^{12}$ Einige dieser Unterschiede sind essenziell für die wissenschaftliche Funktion von Modellen. Dies ist eine zentrale Einsicht philosophischer Studien zu Modellen und Modellierung: wissenschaftliche Modelle funktionieren nicht allein auf Grund ihrer Ähnlichkeiten zu den Zielen, sondern auch auf Grund von Idealisierungen und anderen Abweichungen von diesen Zielen. Es ist allgemein bekannt, dass wissenschaftliche Modelle häufig idealisiert sind; $d$. h., dass sie unrealistische, falsche oder fiktive Elemente enthalten. Auf einige Idealisierungen kann verzichtet werden, andere hingegen tragen zur Nutzbarkeit des Modells als Ersatzstudienobjekt bei. ${ }^{13}$ Insbesondere ist ein Modell im Vergleich zu seinem Ziel einfacher, ermöglicht effizienteres und sparsameres Arbeiten und zeigt die zu untersuchenden Phänomene in zugänglicherer Weise. So sind einige Hirnorganoide beispielsweise so modifiziert, dass sie transparent sind, um eine bessere Visualisierung und Datensammlung interner Prozesse zu ermöglichen (Knoblich, 2020). Dies macht sie In-vivo-Organen unähnlich, aber effektiver als Modelle derselben. Einer der großen Vorteile von Organoiden als Modellen ist ihre Verfügbarkeit für viele Formen der Datenerhebung - wir können gerade deshalb so viel von ihnen lernen, weil sie ex vivo (außerhalb des Körpers) vorliegen als sichtbare und handhabbare Versuchsobjekte. Ihre relative Einfachheit und Zugänglichkeit ermöglicht die Anwendung experimenteller Methoden, die in komplexeren und weniger gut zugänglichen Systemen, d. h. in menschlichen Organen in vivo, nicht möglich (oder ethisch nicht vertretbar) sind. Essenziell für ihre Modellfunktion ist, dass Organoide kleiner und strukturell weniger komplex sind und nicht alle Funktionen des vollentwickelten menschlichen Organs aufweisen. Anstelle einer perfekten Nachahmung von In-vivo-Prozessen zeigen sie bestimmte Aspekte

12 Teller (2001) bietet überzeugende philosophische Argumente dafür, dass „ein perfektes Modell“, das mit seinem Ziel in jeglicher Hinsicht übereinstimmt, ein Widerspruch in sich selbst wäre. Dasselbe Argument findet sich in phantasievoller, aber nicht weniger scharfsinniger Form, bei Borgés (1946).

13 Bolker (1995) gibt eine aufschlussreiche, kritische Darstellung dieser Unterschiede in klassischen Modellorganismen der Entwicklungsbiologie. 
der Organentwicklung in vereinfachter Form, ohne die vollständige physiologische Organisation und funktionelle Integration in heranwachsenden Säugetieren.

Dieser konzeptuelle Punkt hat mehrere praktische Implikationen. Erstens hat das Projekt, Organoide ihren Zielen ähnlicher zu machen, notwendigerweise Grenzen. Das bedeutet nicht, dass jene Projekte verworfen werden sollten, sondern lediglich, dass ihr Erfolg nicht das letztgültige Ziel der Organoidforschung ist. Organoide sind für die Wissenschaft von Wert - nicht trotz, sondern wegen der Idealisierungen, die sie $\mathrm{zu}$ effektiven Surrogaten machen. Zweitens und in Zusammenhang damit, muss der Slogan „Organoide rekapitulieren die menschliche Organentwicklung“ mit Vorsicht interpretiert werden. Die Alltagsbedeutung von „rekapitulieren“ ist ,etwas dieselben Stadien durchlaufend zu wiederholen“. Zu behaupten, dass Organoide „die Entwicklungsschritte, die in vivo vorkommen, rekapitulieren", ${ }^{14}$ bedeutet, buchstäblich zu sagen, dass In-vivo- und Ex-vivo-Prozesse gleich sind; dass sie dieselben Stadien durchlaufen. Es bestehen zwei Probleme mit dieser Aussage. Erstens ist unser Zugang zum In-vivo-Prozess sehr beschränkt; wir wissen aus direkten Untersuchungen nicht viel darüber. Ein Großteil unseres Wissens über die menschliche Entwicklung beruht auf Analogieschlüssen von Modellorganismen oder neuerdings von Zellkultur- und Tissue-Engineering-Systemen (unter denen Organoide eine wichtige Klasse bilden). Dass die Organentwicklung in vivo und ex vivo dieselben Stadien durchläuft, ist kein einfaches Faktum, das auf direkter Beobachtung der beiden Prozesse im Vergleich basiert. Es ist, zumindest in den meisten Fällen, eine Schlussfolgerung oder eine Arbeitshypothese. Zweitens bestehen, wie gerade erörtert, gute Gründe für die Annahme, dass die Entwicklungsprozesse in vivo und ex vivo nicht strikt identisch sind, sondern einander in bestimmter Hinsicht ähnlich, in anderer unähnlich sind. Einige Unähnlichkeiten sind Idealisierungen, die Organoide zu guten Modellen machen - einfach, handhabbar, zugänglich, sehr genau experimentell kontrollierbar. Einige Abweichungen der Modelle von ihren Zielen machen sie nützlicher und zugleich weniger realistisch. Dem Begriff „rekapitulieren“ entgeht dieser Aspekt der Modellierung. Obgleich Organoidforschende sich dieser Unterschiede und Qualifizierungen sehr bewusst sind, lädt die Behauptung, dass „Organoide die menschliche Organentwicklung rekapitulieren“ zu Missverständnissen bei einem breiteren Publikum ein.

14 Das Zitat ist eine Paraphrase von Huch et al. (2017: 939); es gibt viele weitere Beispiele. 


\subsection{Generative Modelle: Stammzellen und Organoide}

Als nächstes betrachte ich den generativen Aspekt von Organoiden, da er zu ihrer Modellfunktion gehört. Wissenschaftliche Modelle sind, wie oben erwähnt, ontologisch vielfältig. Manche von ihnen sind Lebewesen; Modellorganismen sind das bekannteste Beispiel. Stammzelllinien sind auch „lebende Modelle“ (Fagan, 2013 und 2016). Dies hat einige interessante Implikationen für die Organoidforschung, weil Organoide per definitionem aus Stammzellen hergestellt werden. ${ }^{15}$ Genauer gesagt werden Organoide durch die generativen Entwicklungsfähigkeiten von Stammzellen produziert. Um die Rolle von Organoiden als Modelle zu verstehen, müssen wir untersuchen, wie sie gemacht werden; wie es dazu kommt, dass sie Merkmale aufweisen, die denen von In-vivo-Organen ähneln. Damit kommen wir zu Stammzellen.

Stammzellen werden „funktionell darüber definiert, dass sie die Fähigkeit zur Selbsterneuerung und zum Hervorbringen differenzierter Zellen haben“ (Melton, 2013: 7). ${ }^{16}$ Das heißt, dass Stammzellen in Bezug auf zwei zentrale biologische Prozesse definiert werden: Reproduktion und Entwicklung. Es gibt viele Arten von Stammzellen. Aber Stammzellen werden, im Unterschied $\mathrm{zu}$ anderen Zelltypen, funktionell definiert, nicht in Bezug auf ihre Struktur, Morphologie, Physiologie, Genexpression etc. (Natürlich haben alle Zellen solche Merkmale, aber diese liegen der Definition von Stammzellen nicht zugrunde.) Stammzellen werden durch das definiert, was sie tun: (i) sich teilen, um Nachkommen hervorzubringen, die auch Stammzellen sind (Selbsterneuerung ${ }^{17}$ ), und (ii) sie generieren Nachkommenzellen, die auf bestimmte Funktionen innerhalb des sich entwickelnden Organismus spezialisiert sind (Differenzierung). Das Konzept der Differenzierung ist ziemlich komplex und bezieht sich auf Änderungen der Zelleigenschaften, die während des Entwicklungsprozesses des Organismus auftreten. Grob gesagt sind Zellen mehr oder weniger differenziert je nach

15 Simian und Bissell (2017) schlagen eine umfassendere, historisch informierte Definition vor, die die Modellierungsfunktion von Organoiden betont, aber auch andere zelluläre Quellen als Stammzellen zulässt. Obgleich die auf Stammzellen eingeschränkte Definition gegenwärtig am prominentesten erscheint und in diesem Kapitel vorausgesetzt wird, heben Simian und Bissell zu Recht den konzeptuellen und technischen Beitrag früherer, von der Stammzellforschung unabhängiger Forschungsprogramme an Gewebekulturen hervor.

16 Im Wesentlichen dieselbe Definition findet sich im Glossar der Internationalen Gesellschaft für Stammzellforschung (2016) und des Europäischen Stammzellnetzwerks (2016), dem Stammzellglossar des NIH (Gesundheitsamt) und anderen prominenten Schauplätzen der Stammzellforschung.

17 Der Begriff „Selbsterneuerung“ ist etwas irreführend; Stammzellen bringen nicht buchstäblich sich selbst hervor durch Zellteilung. Sie bringen vielmehr Nachkommen hervor, die als Stammzellen derselben Art klassifiziert werden. Stammzellen sind selbsterneuernd auf der Populationsebene. 
ihrer Position in der Entwicklungstrajektorie auf Zellebene. Diese Trajektorie (Entwicklungsbahn) endet mit den „reifen“ (vollentwickelten) Zelltypen, aus denen der Körper eines erwachsenen Organismus zusammengesetzt ist; die Endpunkte des Entwicklungsprozesses auf Zellebene. Jede Art von Stammzellen vermag eine bestimmte Bandbreite an differenzierteren Zelltypen hervorzubringen. Diese Bandbreiten werden in Form von allgemeinen Kategorien des Potenzials von Stammzellen erfasst: totipotente Stammzellen können einen ganzen Organismus und umgebende Membranen hervorbringen; pluripotente Stammzellen können alle Zelltypen des vollentwickelten Organismus hervorbringen; multipotente Stammzellen können einige, aber nicht alle Zelltypen des vollentwickelten Organismus hervorbringen - üblicherweise beschränkt auf eine Gewebeart.

Organoide werden aus drei Arten von Stammzellen gewonnen: embryonalen Stammzellen, induzierten pluripotenten Stammzellen und gewebespezifischen (adulten) Stamm-/Vorläuferzellen. ${ }^{18}$ Die ersten beiden Arten sind pluripotent und weisen eine unbegrenzte Selbsterneuerungsfähigkeit auf; die dritte Art ist multipotent und weist typischerweise eine zeitlich begrenzte Selbsterneuerungsfähigkeit auf (auch wenn Zellkulturbedingungen dem entgegenwirken können). Humane embryonale Stammzellen (hES-Zellen) werden aus der inneren Zellmasse eines sehr frühen (ungefähr 5 Tage alten) menschlichen Embryos gewonnen. Extrahierte Zellen werden in eine zweidimensionale künstliche Kultur gegeben, in der spezifische chemische Faktoren durch Schichten von „Feeder“-Zellen (die der Ernährung dienen) oder chemisch definierten Nährmedien bereitgestellt werden. Zellen, die sich unter diesen Bedingungen schnell teilen, bilden Kolonien, die auf zweidimensionalen Kulturplatten wachsen. Schnell wachsende Kolonien (d. h. solche, die eine schnelle Selbsterneuerung aufweisen) werden ausgewählt und alle paar Wochen auf neue Kulturplatten versetzt. Dadurch erhält man eine Abstammungslinie selbsterneuernder, undifferenzierter Zellen: eine Stammzelllinie. HES-Zellkulturen sind zweidimensional; die Zellen teilen sich und wachsen so, dass sie, gebadet in flüssiger Zellkulturlösung, eine flache Ebene bedecken. Entscheidend ist, dass diese Zellkulturlösung biochemische Faktoren enthalten muss, die eine Differenzierung blockieren. In einer anderen Umgebung käme es zur Differenzierung. Pluripotente hES-Zellen können, die richtige Umgebung vorausgesetzt, in jeden Zelltyp des menschlichen Körpers differenzieren. Welcher

18 Der Einfachheit halber liegt der Fokus hier auf humanen Organoiden und Stammzellen. Es gibt auch eine große Vielzahl an Organoiden, die aus Mausstammzellen gewonnen wurden; die Hauptpunkte dieses Kapitels gelten für diese ebenfalls, wenn auch die experimentellen Ableitungen etwas anders sind. 
Entwicklungspfad eingeschlagen wird (z. B. Nerven, Knochen, Blut etc.), hängt von der spezifischen Zellkulturumgebung ab.

Humane induzierte pluripotente Stammzellen (hiPS-Zellen) ähneln hES-Zellen in ihren Fähigkeiten zur Selbsterneuerung und Differenzierung. Sie haben jedoch einen ganz anderen Ursprung im Organismus. Gewonnen werden hiPS-Zellen durch Extrahierung differenzierter Zellen aus einem Teil des Körpers eines post-embryonalen Organismus (gewöhnlich aus der Haut; aber alle Zellen, die unter Kulturbedingungen erhalten werden können, sind verwendbar). Diese extrahierten Zellen werden in eine zweidimensionale Kultur gesetzt, die 2-4 spezifische Gene enthält, die Transkriptionsfaktoren (TF) kodieren. TF sind Proteine, die spezifisch an bestimmte Sequenzen der DNA binden, was wiederum Auswirkungen auf die Genexpression in der Nähe des Chromosoms hat. Deshalb kann die Veränderung der Expression eines TF-Gens einen Unterschied machen für die Expression dutzender, ja hunderter anderer Gene - einschließlich anderer TF-Gene. TF-Proteine sind demzufolge phänotypische Schalter, die umfangreiche Genexpressionsmuster innerhalb einer Zelle koordinieren können. HiPS-Zellen sind das Ergebnis einer solchen Veränderung. Nach wenigen Wochen transformieren sich einige dieser kultivierten Zellen (ca. 0,5\% in frühen Experimenten) so, dass sie embryonalen Stammzellen in Hinblick auf Morphologie und Entwicklungsvermögen ähneln. Zellen, die sich unter diesen Bedingungen teilen und selbsterneuern, initiieren eine Zelllinie von hiPS-Zellen. Die Transformation zu hiPS-Zellen wird als „Reprogrammierung“ bezeichnet (Takahashi/Yamanaka, 2006). Unterschiedliche Spezifizierungen der grundlegenden Methode sind mehr oder weniger effektiv in der Herstellung von hiPS-Zellen, die ihrerseits hinsichtlich ihrer molekularen Eigenschaften und ihres Entwicklungsvermögens (in häufig subtiler Weise) variieren.

Gewebespezifische (adulte) Stammzellen haben eingeschränktere Fähigkeiten zur Selbsterneuerung und Differenzierung und tendieren zur Produktion von Organoiden, die vollentwickelten Organen besser entsprechen (Yin et al., 2016; Munsie et al., 2017; Takebe/Wells, 2019). Es gibt viele verschiedene Arten gewebespezifischer Stammzellen, die jeweils aus einem bestimmten Organ oder Gewebe eines post-embryonalen Organismus isoliert wurden. Am besten charakterisiert sind Stammzellen des Blutes und des Immunsystems: hämatopoetische, d. h. blutbildende Stammzellen. Viele weitere gewebespezifische Stammzellen, einschließlich Nerven-, Darm- und Muskelstammzellen, sind ebenfalls charakterisiert worden. Wie bei hiPS-Zellen ist der Ursprungsorganismus gewebespezifischer Stammzellen kein Embryo, sondern ein weiter entwickelter Organismus. Im Unterschied zu hiPS-Zellen ist jedoch nicht jeder Zelltyp geeignet. Gewebespezifische Stammzellen werden aus einem Organ oder Gewebe des Organismus isoliert, gewöhnlich in unvollkommener Weise und verbunden mit einiger 
Spekulation. Die Herausforderung besteht darin, reine Populationen aller und nur der Stammzellen eines vorhandenen Gewebes oder Organs zu isolieren (Fagan, 2013). Sind sie einmal identifiziert, werden gewebespezifische Stammzellen für gewöhnlich ziemlich schnell gemessen oder verwendet; erst kürzlich sind längerfristige Kulturverfahren für gewebespezifische Stammzellen entwickelt worden (und nur für einige Organe/Gewebe). Letztere Verfahren sind allerdings notwendig, um Organoide herzustellen.

Ein klares Ergebnis dieses Überblicks ist, dass organoidproduzierende Stammzellen ihrerseits aus multizellulären Organismen gewonnen werden. Dies ist der Fall bei allen Stammzellen - darüber hinaus bestimmen die Spezieszugehörigkeit des Ursprungsorganismus, das Entwicklungsstadium und die Stelle der Zellgewinnung wichtige Eigenschaften einer kultivierten Stammzelllinie. Bei embryonalen und gewebespezifischen Stammzellen hängt der Grad der Fähigkeit zur Selbsterneuerung und Differenzierung vom Zeitpunkt der Zellentnahme und ihrem Ort im Organismus ab. Die experimentelle Methode der Induzierung von Pluripotenz überwindet diese Abhängigkeiten. Aber auch dann behalten hiPS-Zellen die Spezieszugehörigkeit und das individuelle Genom ihres Ursprungsorganismus. Eine kontinuierliche ,Reihe (,lineage") von Experimenten“ verbindet jedes Organoid mit einer Stammzelllinie und dadurch mit einem Ursprungsorganismus. Dies stellt eine gewisse materielle Kontinuität zwischen dem Ursprungsorganismus, der Stammzelllinie und dem Organoid sicher. In Verbindung mit dem für Stammzellen charakteristischen generativen Entwicklungsvermögen schafft diese materielle Kontinuität zentrale Ähnlichkeiten zwischen Organoiden und ihren Zielen: menschlichen Organen. Wichtige genetische, molekulare und zelluläre Komponenten menschlicher Organismen werden buchstäblich in Organoide eingebaut. Obwohl diese Kontinuitäten nicht garantieren, dass sich Organoide durch dieselben oder ähnliche Prozesse wie In-vivo-Organe entwickeln, bilden sie doch eine Grundlage für Schlussfolgerungen auf solche Ähnlichkeiten; prima facie stützen sie modellbasierte Schlussfolgerungen in Bezug auf das Ziel. Bei Organoiden, die viele derselben molekularen, genomischen und zellulären Komponenten enthalten wie der Ursprungsorganismus, kann vernünftigerweise erwartet werden, dass sie diese Komponenten während der Entwicklung in ähnlicher Weise kombinieren. Stammzellen sind entscheidend für diese Kontinuität und so auch für diese Art der Stützung modellbasierter Schlussfolgerungen bei Organoiden.

Die Entwicklungsfähigkeiten von Stammzellen beeinflussen die Modellfunktion von Organoiden auch in anderer Hinsicht. Man ist leicht verleitet, jede Art von Organoid als individuell auf das entsprechende menschliche Organ bezogen zu konzeptualisieren und zu bewerten. Im Sinne der obigen philosophischen Analyse läuft dies darauf 
hinaus, Modell und Ziel als feststehend zu betrachten und zugleich mehrere Nutzer und Zwecke zuzulassen. Diese Konzeption von Organoiden eröffnet die Möglichkeit von Abwägungen zwischen den Zwecken. Für den Organersatz konstruierte Organoide beispielsweise wären am besten, wenn sie menschlichen Organen so wirklichkeitsnah wie möglich entsprächen. Hingegen sollten Organoide, die für ein effizientes und kostengünstiges Screening in der Medikamentenforschung vorgesehen sind, einfach und leicht handhabbar sein und eine schnelle und eindeutige Messung der Ergebnisse ermöglichen. Leicht ließen sich noch mehr Beispiele finden; ein Grund, warum Organoide so viel Aufmerksamkeit erhalten haben, ist die Fülle ihrer potenziellen Anwendungen. Viele davon erfordern eine große Ähnlichkeit zu menschlichen Organen - aber nicht alle und nicht immer dieselben Ähnlichkeiten. Wenn also Organoide so konzeptualisiert werden, dass sie menschlichen Organen eins $\mathrm{zu}$ eins entsprechen, erscheinen Kompromisse hinsichtlich der Merkmale, die für andere Anwendungen erforderlich sind, unvermeidbar.

Solch eine „ein Organoid, ein Organ“-Sichtweise wird jedoch durch die Verbindung von Organoiden mit Stammzellen untergraben. In früheren Arbeiten argumentiere ich, dass Stammzelllinien und ihre Entwicklungsprodukte als Modellgefüge (,fabric of models") konzeptualisiert werden sollten, aus dem Erkenntnisse über die menschliche Entwicklung stückweise hervorgehen (Fagan, 2013 und 2016). Die menschliche Entwicklung ist ein äußerst komplexes Phänomen, das kein einzelnes handhabbares Modell adäquat repräsentieren kann. Stammzellen sind nicht nur leistungsfähige Werkzeuge für die Modellierung von Aspekten der Entwicklung, sondern auch zur Generierung anderer Modelle - einschließlich, aber nicht ausschließlich, von Organoiden. Auf diese Weise ahmt die Modellfunktion von Stammzellen ihre Entwicklungsfähigkeiten nach: Stammzellen sind Modelle, die andere Modelle generieren, genauso wie (und weil) Stammzellen Zellen sind, die durch Differenzierung andere Zellarten generieren. Diese Behauptung mag mysteriös erscheinen. Um sie zu demystifizieren, müssen wir die experimentellen Methoden zur Messung der Differenzierungsfähigkeit von Stammzellen betrachten. Mit diesen Methoden werden Proben von Stammzelllinien dazu gebracht zu differenzieren, indem man die Proben in eine geeignete Kulturumgebung versetzt. Es gibt eine Vielzahl an Variationen dieses allgemeinen Methodenschemas, die ich hier kurz durchgehe.

Eine Methode sind Experimente der "gerichteten Differenzierung“: Proben einer Stammzellinie werden in zweidimensionale Kulturen gebracht, die biochemische Faktoren enthalten, welche eine Differenzierung entlang mehrerer verschiedener Entwicklungspfade anregen. Für pluripotente Stammzellen sollten alle Hauptentwicklungspfade vorhanden sein: Blut, Knochen, Leber, Neuronen etc. Für gewebespezifi- 
sche Stammzellen ist eine geringere Bandbreite an Ergebnissen relevant. Bei jedem Experiment gerichteter Differenzierung ist das Ergebnis eine Reihe an Schichten von Populationen bestimmter vollentwickelter Zelltypen, die zeigen, dass das Potenzial einer bestimmten Stammzelle die Bildung dieser Zelltypen einschließt. Auch wenn es sich dabei um eine gute Methode zur Generierung großer Populationen eines bestimmten Zelltyps handelt, sind Experimente gerichteter Differenzierung dennoch kein effizienter Weg, um auf Pluripotenz zu testen. Stattdessen wird das Entwicklungspotenzial einer vorhandenen Stammzelllinie üblicherweise dadurch bestimmt, dass man experimentelle Stellvertreter (,proxies“) generiert und testet. Die beiden gebräuchlichsten Proxies sind „embryoid bodies“ (embryo-ähnliche Körper) und Teratoma: dreidimensionale Zellkulturprodukte, die jeweils äußerst einfach und hochgradig desorganisiert sind. Embryoid Bodies werden aus kultivierten pluripotenten Stammzellen hergestellt, die in einer flüssigen Lösung treiben. So schweben sie frei von ihren zweidimensionalen Kolonien im Nährmedium und organisieren sich zu Kugeln von 0,1-0,2 mm Durchmesser. Diese bestehen aus einem inneren Kern undifferenzierter Stammzellen, der von einer Schicht weiter differenzierter Zellen umhüllt ist. Diese einfachen Zellstrukturen ähneln einem frühen Stadium der Embryonalentwicklung von Säugetieren. Teratoma werden dadurch hergestellt, dass menschliche Stammzellen in einen durch Inzucht erzeugten und dadurch genetisch homogenen Mausstamm gegeben werden, worin die Zellen nicht abgestoßen werden, sondern zu Tumoren in den Mäusen heranwachsen. Die entstandenen Tumoren werden entnommen, seziert und histologisch analysiert, um die Bandbreite an Zelltypen zu bestimmen, die sich aus den injizierten Stammzellen entwickelt haben. Diese Tumoren bestehen aus verschiedenen spezialisierten Zellen und Geweben, doch fehlen ihnen alle sonstigen Aspekte der menschlichen Entwicklungsorganisation. Organoide sind eine andere Art Entwicklungsprodukt von Stammzellen - komplexer als Embryoid Bodies und im Vergleich zu Teratomen weiter entwickelt in ihrer Organisation. ${ }^{19}$ Aber Organoide werden, genau wie ihre experimentellen Proxies, durch die gezielte Veränderung der Umgebung der Stammzellen und ihrer Derivate hergestellt.

Statt Organoide als isolierte Modelle zu konzeptualisieren, die jeweils ein menschliches Organ repräsentieren, sollten wir Organoide als ein breiteres Gefüge von Modellen („fabric of models“) verstehen. Die unterschiedlichen Modelle, aus denen sich

19 Embryo-ähnliche Strukturen (Gastruloide) werden ebenfalls aus Stammzellen hergestellt, dadurch dass man zweidimensionale Stammzellen in spezifischen geometrischen Mustern wachsen lässt. Viele Argumente/Punkte, die hier über Organoide gemacht werden, gelten auch für diese synthetischen Modell-Embryonen. 
dieses Gefüge zusammensetzt - Zellpopulationen, Embryoid Bodies, Teratome, Organoide, und mehr - werden alle aus Stammzellen durch deren außergewöhnliche Entwicklungsfähigkeiten gewonnen. Das Entwicklungspotenzial von Stammzellen wird, wie oben diskutiert, entsprechend der Bandbreite an Zelltypen definiert, die eine bestimmte Stammzelle hervorbringen kann, eine geeignete Umgebung vorausgesetzt. Aber die experimentellen Methoden, die zum Einsatz kommen, um dieses Potenzial, insbesondere der Pluripotenz, zu messen, zeigen, dass die Entwicklungsfähigkeiten von Stammzellen nicht auf die Zellebene begrenzt sind. Stattdessen realisiert jedes dieser experimentellen Produkte die Entwicklungsfähigkeiten von Stammzellen in etwas unterschiedlicher Weise. (Ich bezeichne diese Erweiterung des Stammzellpotenzials als „Entwicklungs-Versatilität“.) Nun könnten wir all diese verschiedenen Entwicklungsprodukte von Stammzellen als ungeordnete, verwirrende Ansammlungen oder Haufen konzeptualisieren. Aber es ist nützlicher, sie so zu begreifen, dass sie (Embryoid Bodies, Organoide, Teratoma und alle anderen) unterschiedliche Aspekte eines sehr komplexen Phänomens zum Ziel haben. Dieses Phänomen ist, natürlich, die Entwicklung des Organismus (speziell des menschlichen). Die aus Stammzellen erzeugten experimentellen Produkte beleuchten jedes einen anderen Aspekt der Organismusentwicklung in vivo, und unser Verständnis des Phänomens wird dadurch verbessert, dass all diese unterschiedlichen Modelle zusammen betrachtet werden.

In früheren Arbeiten habe ich erläutert, dass dieses Arrangement unterschiedlicher stammzellbasierter Modelle die Palette unterschiedlicher Modi der Differenzierung repräsentiert, die an der Entwicklung eines Organismus beteiligt sind (Fagan, 2016, 2017, 2018). Mindestens sechs solcher Modi lassen sich unterscheiden, von denen jeder einen anderen Aspekt des Prozesses der Entstehung eines multizellulären Organismus aus undifferenzierten Zellen beleuchtet: einfach, aufgelöst, zellulär, organähnlich und embryo-ähnlich; alle sind integriert in den In-vivo-Prozess der normalen Entwicklung eines Organismus. Organoide (organähnliche) Entwicklung ist lediglich einer dieser Modi: Die Kombination dreidimensionaler Positionierung und spezifischer Signale produziert zahlreiche spezialisierte Zelltypen, die sich der dreidimensionalen und funktionalen Organisation eines bestimmten Organs annähern (approximieren). Kein einzelnes Stammzellderivat erfasst jeden bedeutenden Aspekt der menschlichen Entwicklung. Stattdessen ergänzen die verschiedenen Modelle einander und beleuchten unterschiedliche Aspekte eines sehr komplexen In-vivo-Prozesses.

Dasselbe gilt, wenn wir die Vielfalt der Organoide betrachten. Die Entwicklung auf Organebene ist kein einzelner Prozess, sondern selbst komplex und diversifiziert. Darin involviert sind Muster von Zellbewegungen und die Bildung einer Grenze zwischen Organismus und Umgebung, das weitere organismische Milieu. Jede Art Organoid ver- 
körpert einige Aspekte dieses In-vivo-Prozesses, andere hingegen nicht. Daraus ergibt sich, dass anstelle von „einem Organoid für ein Organ“ zahlreiche verschiedene Organoidmodelle auf unterschiedliche Aspekte der Entwicklung eines Organs abzielen. ${ }^{20}$

\subsection{Selbstorganisation und Selbstassemblierung: im Kontext}

Organoide werden oft als im Besitz einer Fähigkeit zur „Selbstorganisation“ oder „Selbstassemblierung“ beschrieben. ${ }^{21}$ Eine Common-Sense-Interpretation dieser Aussagen ist, dass Organoide sich durch einen internen, zellgesteuerten Prozess bilden; durch so etwas wie ein vorgegebenes Programm zur Organbildung. Dies ist jedoch inkonsistent mit dem, was wir über Stammzellen (zumindest in Säugetieren) wissen. Wie oben erläutert, sind Stammzellen versatil (vielseitig) in ihrer Entwicklung - sie vermögen verschiedene Modi der Entwicklung zu realisieren in Abhängigkeit von ihrer Umweltumgebung. Welcher Entwicklungsmodus von einer Stammzelle realisiert wird, wird durch Eigenschaften der Umgebung dieser Zelle und der Umgebung ihrer Nachkommen bestimmt. ${ }^{22}$ Allgemeiner gesagt, ist die Identität von Stammzellen einschließlich der Modi ihrer Entwicklung - kontextabhängig. Experimente, die das Entwicklungspotenzial aufzeigen, nutzen diese Eigenschaft, indem sie Stammzellen zur Differenzierung in spezifischer (und unterschiedlicher) Weise anregen, durch Veränderung der Geometrie, biochemischen Komposition oder zellulären Zusammensetzung ihrer Umgebung. Es ist seit Langem bekannt, dass „der Stammzell-Phänotyp keineswegs festgelegt zu sein scheint“, sondern dass „das Verhalten von Stammzellen von ihrer Mikro-Umgebung kontrolliert wird“ (Potten/Lajtha, 1982: 454; Yin et al., 2016: 27). Über 50 Jahre Stammzellexperimente zeigen, dass die Stammzellidentität überaus empfindlich auf Eigenschaften der lokalen Mikro-Umgebung reagiert, sowohl in vivo als auch in vitro. In der Tat ist die Ausnutzung dieser Kontextabhängigkeit das grundlegende Designprinzip aller Stammzellexperimente: Stammzellkandidaten werden in eine neue Umgebung versetzt und ihre Charakteristika oder die ihrer Nachkommen

20 Dies könnte auch einen Weg zum Umgang mit Variabilität (Mangel an Reproduzierbarkeit) in gegenwärtigen Organoidmodellen hinsichtlich Größe, Zelltypen und der relativen Positionierung von Geweberegionen eröffnen.

21 Z. B. Organoide „fügen sich selbst zusammen (self-assemble), um die zelluläre Organisation des Organs (selbst) zu bilden“ (Lancaster/Knoblich, 2014: 283); siehe auch Nature Methods (2018: 1).

$22 \mathrm{Zu}$ diesen Eigenschaften gehören die An- oder Abwesenheit bestimmter biochemischer Signale, die räumliche/geometrische Anordnung der Zellen, physikalische Grenzen des Systems und physikalische Faktoren wie z. B. Sauerstoffgehalt und pH-Wert (siehe unten). 
werden in diesem Kontext gemessen. Diese grundlegende Experimentalstrategie wird weiterhin verfolgt, sie enthüllt stückweise die unterschiedlichen Faktoren, die in die Entstehung der Organisation eines Organismus aus einer Stammzelle involviert sind.

Die Organoidforschung ist Teil dieses größeren Unterfangens. Ihr näheres Ziel ist es, zu verstehen, wie Organoide sich aus einer Stammzellpopulation selbst organisieren. Obwohl unser Wissen über diesen Prozess unvollständig ist, haben wir bereits eine Menge durch die Organoidforschung gelernt. Im Allgemeinen zeichnet sich die folgende Sichtweise ab: Stammzellen werden auf dreidimensionale Arrangements "gesät“, die Interaktionen zwischen den Zelloberflächenmolekülen auslösen. Im Zusammenspiel mit physikalischen Kräften und biochemischen Faktoren legen diese Interaktionen die Zellen darauf fest, sich entlang bestimmter Pfade zu entwickeln und eine bestimmte Position zueinander einzunehmen („cell sorting out“). In diesen Exvivo-Gewebekulturumgebungen stehen verschiedene Faktoren in dynamischer Wechselwirkung miteinander und mit Stammzellen auf verschiedenen räumlich-zeitlichen Ebenen: „Stützzellen“ („support cells“), die Signalfaktoren absondern, die extrazelluläre Matrix, mechanische Kräfte, Sauerstoffgehalt, pH-Wert und mehr (Yin et al., 2016: 28). Insofern ist Selbstorganisation, wie sie derzeit verstanden wird, ein Muster dynamischer Veränderung in einem komplexen Netzwerk interagierender Faktoren. Selbstorganisation erklärt sich nicht als Prozess, der durch ein vorhandenes, zellinternes Programm gesteuert würde, sondern als dynamisches Zusammenspiel zellinterner Mechanismen mit zahlreichen interagierenden Umweltfaktoren.

Warum also schreiben Organoidforschende die Fähigkeit zur Bildung von Organoiden dem „Selbstorganisations“-Potenzial von Stammzellen zu? Die Begriffe „Selbstorganisation“ und „Selbstassemblierung“ markieren keinen Unterschied zwischen Zellen und ihren Umgebungen, sondern zwischen dem, was Versuchsleitende in Bezug auf die Entwicklung von Organoiden kontrollieren können und dem, was sie nicht kontrollieren können. Organoide weisen Selbstorganisation relativ zum erwarteten, vorhersagbaren Verhalten von unter anderen Umweltbedingungen kultivierten Stammzellen auf. Nicht wir kontrollieren den organ-ähnlichen Entwicklungsprozess; die Zellen (unter diesen spezifischen Bedingungen) tun dies. ${ }^{23}$ „Selbstorganisation“ und „Selbstassemblierung“ von Organoiden bringt unser unvollständiges Wissen und unsere unvollständige Kontrolle dieses Prozesses zum Ausdruck - diese Grenzen zu überwinden ist ein wichtiges Ziel der Organoidforschung. Eine große Vielfalt an Stammzellexperimen-

23 Z. B. ist es „häufig schwierig, den Zelltyp, die Organisation sowie die Zell-Zell- oder Zell-MatrixWechselwirkungen zu kontrollieren [...] Organoidbildung ist in hohem Maße abhängig von zellautonomer Selbstorganisation, die sich noch nicht einfach kontrollieren lässt" (Yin et al., 2016: 25-26). 
ten hat uns gezeigt, wie sich Stammzelleigenschaften durch Eigenschaften der zellulären Umgebung kontrollieren lassen; die Reprogrammierung vollentwickelter Zellen zu hiPS-Zellen ist ein eindrucksvolles Beispiel. Aber in Organoiden ist unsere Kontrolle unvollkommen aufgrund bestehender Lücken in unserem Wissen über organ-ähnliche Entwicklung von Stammzellen. Sobald das Forschungsfeld das angestrebte Niveau der Kontrolle über und Rückschlüsse auf die menschliche Organentwicklung erlangt, dürfte der Begriff „Selbstorganisation“ wegfallen. ${ }^{24}$ Zum jetzigen Zeitpunkt sollte die Selbstorganisation und Selbstassemblierung von Organoiden nicht als Zeichen dafür gewertet werden, dass die Organoidorganisation irgendwie in Stammzellen „,vorprogrammiert“ sei, um Missverständnissen vorzubeugen. Stammzellen „selbst-assemblieren“ zu einem Modellorgan nur unter sehr spezifischen Bedingungen - von denen wir einige verstehen, während andere unbekannt bleiben.

\subsection{Schlussfolgerungen}

Hier fasse ich die zentralen Aussagen dieses Kapitels zusammen. Erstens klärt eine einflussreiche philosophische Darstellung von Organoiden deren wissenschaftliche Bedeutung als Modellorgane, d. h. als Ex-vivo-Stellvertreter für menschliche Organe in vivo. Als Modelle stützen Organoide Schlussfolgerungen über ihre In-vivo-Gegenstücke auf Basis bestimmter empirisch verifizierter Ähnlichkeiten mit ihnen: kompositionelle, strukturelle und funktionelle. Aber diese Ähnlichkeiten sind nicht perfekt oder vollständig; Modelle gewähren auch aufgrund von Idealisierungen Wissen über ihre Ziele. Fortschritt in der Organoidforschung ist nicht einfach eine Frage der technischen Herstellung größerer Ähnlichkeit zu In-vivo-Organen. Philosophische Erkenntnisse über Modelle legen eine nuanciertere Sicht nahe: Organoide sind so konstruiert, dass sie In-vivo-Organen in bestimmten spezifischen Hinsichten ähneln, in Graden, die notwendigerweise nicht an eine perfekte Ähnlichkeit heranreichen. Organoide sind gerade deshalb wertvolle Modelle, weil sie intermediäre Kultursysteme sind - sehr viel einfacher als das Original, aber komplexer als andere Zellkulturmodelle.

24 Variabilität zwischen Organoiden, die nach derselben Methode hergestellt wurden (und damit das Problem des Mangels an Reproduzierbarkeit) dürfte durch die angestrebten Formen experimenteller Kontrolle bzw. der Kontrolle durch Experimentatoren beseitigt werden. Die bei Park (2019) diskutierten verschiedenen Herausforderungen für die Organoidforschung sind alle Aspekte solcher Kontrolle: Kontrolle der 3-D-Kulturmikroumgebung; dynamische Wechselwirkungen zwischen Geweben und Organen; und Reduzierung der Variationen hinsichtlich der Größe, Struktur, Funktion und Genexpression (960). 
Zweitens werden Organoide aus pluripotenten oder gewebespezifischen Stammzellen hergestellt - was mehrere wichtige Implikationen hat. Organoide verkörpern einige der Entwicklungsfähigkeiten von Stammzellen: speziell die, die an der Organbildung beteiligt sind. Stammzellen können per definitionem andere, von ihnen unterschiedene Lebewesen über einen materiell kontinuierlichen Prozess hervorbringen. Dieses molekulare und zelluläre Material, das an Organoide mittels der Entwicklungsfähigkeiten von Stammzellen weitergegeben wird, ermöglicht Analogieschlüsse vom Modell auf das Ziel. Im weiteren Sinne bilden kultivierte Stammzellen und ihre Entwicklungsprodukte (einschließlich Organoiden) ein Netzwerk komplexer komparativer Beziehungen, die zusammen Einblicke in experimentell weniger gut zugängliche Phänomene von Interesse bieten. Die Konzeptualisierung von Organoiden als Teil dieses größer angelegten Modellgefüges eröffnet Einsichten in ihre Modellfunktion, ihre Grenzen und ihr epistemisches Potenzial. Zum Schluss habe ich die Bedeutung wissenschaftlicher Behauptungen geklärt, dass „Organoide die In-vivo-Organentwicklung rekapitulieren (schrittweise nachbilden)“ und dass „Organoide sich selbst organisieren/assemblieren“, um möglichen Missverständnissen vorzubeugen. Auf diese Weise tragen philosophische Erörterungen von Modellen, generativen Modellen und Stammzellexperimenten dazu bei, den konzeptuellen Hintergrund, die Methodologie und die Ziele der Organoidforschung zu klären.

\subsection{Literaturverzeichnis}

Boesch, B. (2019): The means-end account of scientific, representational actions. In: Synthese 196: 2305-2322.

Bolker, J. A. (1995): Model systems in developmental biology. In: BioEssays 17: 451-55.

Borgés, J.-L. (1946): Del rigor en la ciencia (On exactitude in science).

European Stem Cell Network (2016): Stem cell glossary. Unter: https://www.eurostemcell.org/landin g/explore-stem-cells\#letters [27.05.2020].

Fagan, M. B. (2013): Philosophy of stem cell biology: Knowledge in flesh and blood. Palgrave-Macmillan, London.

Fagan, M. B. (2016): Generative models: human embryonic stem cells and multiple modeling relations. In: Studies in History and Philosophy of Science 56: 122-134.

Fagan, M. B. (2017): Stem cell lineages: between cell and organism. In: Philosophy and Theory in Biology 9, Special Issue: Ontologies of living beings. Unter: https://quod.lib.umich.edu/cgi/t/te xt/text-idx?cc=ptb;c=ptb;c=ptpbio;idno=6959004.0009.006;rgn=main;view=text;xc=1;g=ptpbiog [27.05.2020]. 
Fagan, M. B. (2018): Individuality, organisms, and cell differentiation. In: Bueno, O. et al. (Hrsg.): Individuation across experimental and theoretical sciences. Oxford University Press, Oxford: 114136.

Frigg, R./Nguyen, J. (2018): Scientific representation. In: Zalta, E. N. (Hrsg.): The Stanford Encyclopedia of Philosophy. Unter: https://plato.stanford.edu/archives/spr2020/entries/scientific-represen tation/ [27.05.2020].

Giere, R. N. (1988): Explaining science: A cognitive approach. Chicago University Press, Chicago.

Giere, R. N. (2010): An agent-based conception of models and scientific representation. In: Synthese 172: 269-281.

Godfrey-Smith, P. (2006): The strategy of model-based science. In: Biology and Philosophy 21: 725740.

Hesse, M. (1966): Models and analogies in science. Notre Dame University Press, Notre Dame.

Huch, M. et al. (2017): The hope and the hype of organoid research. In: Development 144: 938-941.

International Society for Stem Cell Research (2016): Stem cell glossary. Unter: https://www.closerloo katstemcells.org/patient-resources/resources-from-isscr/stem-cell-glossary/ [27.05.2020].

Keller, E. F. (2000): Models of and models for: theory and practice in contemporary biology. In: Philosophy of Science 67: S72-S86.

Knoblich, J. A. (2020): Cerebral organoid model reveals excessive proliferation of human caudal late interneuron progenitors in Tuberous Sclerosis Complex. Unter: https://www.stemcell.com/cerebr al-organoids-as-3d-stem-cell-derived-models-of-tuberous-sclerosis-complex.html [17.06.2020].

Lancaster, M. A./Knoblich, J. A. (2014): Organogenesis in a dish: Modeling development and disease using organoid technologies. In: Science 345: 1247125-1-8.

Melton, D. (2013): Stemness: definitions, criteria, and standards. In: Lanza, R./Atala, A. (Hrsg.): Essentials of stem biology. 3. Auflage. Academic Press, San Diego: 7-17.

Munsie, M. et al. (2017): Ethical issues in human organoid and gastruloid research. In: Development 144: 942-945.

National Institutes of Health (2016): Glossary. In: Bethesda, MD (Hrsg.): Stem cell information. Unter: https://stemcells.nih.gov/info/2001report/appendixF.htm [27.05.2020].

Nature Methods Editors (2018): Method of the year 2017: Organoids. In: Nature Methods 15: 1.

Park, S. E. et al. (2019): Organoids-on-a-chip. In: Science 364: 960-965.

Potten, C. S./Lajtha, L. G. (1982): Stem cells versus stem lines. In: Annals of the New York Academy of Sciences 397: 49-61.

Science Editors (2019): Special section: Approximating organs. In: Science 364: 946-965.

Simian, M./Bissell, M. J. (2017): Organoids: A historical perspective of thinking in three dimensions. In: Journal of Cell Biology 216: 31-40. 
Takahashi, S./Yamanaka, S. (2006): Induction of pluripotent stem cells from mouse embryonic and adult fibroblast cultures by defined factors. In: Cell 126: 663-676.

Takebe, T./Wells, J. M. (2019): Organoids by design. In: Science 364: 956-959.

Teller, P. (2001): Twilight of the perfect model model. In: Erkenntnis 55: 393-415.

Weisberg, M. (2013): Simulations and similarity. Oxford University Press, Oxford.

Yin, X. et al. (2016): Engineering stem cell organoids. In: Cell Stem Cell 18: 25-38. 